\title{
Migration of an uncovered self-expandable metal biliary stent in a patient with inoperable cholangiocarcinoma: A case report
}

\author{
Edward Robert John Walton, Ewan Simpson, Mark Callaway
}

\begin{abstract}
Migration of plastic and covered-metal stents is a recognized phenomenon but is an extremely uncommon complication of uncovered selfexpandable metal stents (USEMS). To our knowledge, this is the first case report describing the migration of an uncovered self-expanding metal stent used to treat proximal biliary obstruction secondary to cholangiocarcinoma. This report outlines the complications surrounding this case and discusses the existing literature and the potential complications of uncovered and covered self-expandable metal stents.
\end{abstract}

Keywords: Biliary, Cholangiocarcinoma, Migration, Uncovered self-expanding metal stent (USEMS)

Edward Robert John Walton ${ }^{1}$, Ewan Simpson², Mark Callaway ${ }^{3}$ Affiliations: ${ }^{1}(\mathrm{MbChB}$ FRCR), University Hospitals Bristol, Radiology Department, Bristol Royal Infirmary, Upper Maudlin Street, Bristol, United Kingdom, BS2 8HW; ${ }^{2}$ (MBBS FRCR), University Hospitals Bristol, Radiology Department, Bristol Royal Infirmary, Upper Maudlin Street, Bristol, United Kingdom, BS2 8HW; ${ }^{3}(\mathrm{MRCP}$ FRCR), University Hospitals Bristol, Radiology Department, Bristol Royal Infirmary, Upper Maudlin Street, Bristol, United Kingdom, BS2 8HW.

Corresponding Author: Dr. Edward Robert John Walton, University Hospitals Bristol, Radiology Department, Bristol Royal Infirmary, Upper Maudlin Street, Bristol, United Kingdom, BS2 8HW; Ph: 07752510574; Email: edwardwalton@ hotmail.co.uk

Received: 14 November 2014

Accepted: 10 December 2014

Published: 10 February 2015

\section{How to cite this article}

Walton ERJW, Simpson E, Callaway M. Migration of an uncovered self-expandable metal biliary stent in a patient with inoperable cholangiocarcinoma: A case report. Int $J$ Hepatobiliary Pancreat Dis 2015;5:4-8.

Article ID: 100027IJHPDEW2015

$* * * * * * * * *$

doi:10.5348/ijhpd-2015-27-CR-2

\section{INTRODUCTION}

Biliary stenting is an established treatment option for obstruction of the biliary tree. A randomized control trial by Andersen et al. suggested that endoprosthesis placement is associated with lower total mortality, lower major complications and a reduction in total hospital stay when compared to surgery [1].

Biliary stents can be broadly divided into plastic or metal. Plastic stents are available in a variety of materials, lengths, and designs. Modern metal stents are commonly self-expandable and can be uncovered self-expandable metal stents (USEMS) or may be partially or fully covered self-expandable metal stents (CSEMS). The type of stent selected depends on the aetiology of the obstruction and on patient factors. They are recommended for palliation of malignant hilar strictures where life expectancy is over three months because SEMS are more likely to remain patent for longer than plastic stents.

We present a case of a 52-year-old female with hilar cholangiocarcinoma causing jaundice that underwent palliative stenting with an USEMS. The case was complicated by distal migration resulting in re-obstruction necessitating repeat intervention. Stent migration is a recognized complication with both plastic 
stents and CSEMS. It is seen extremely uncommonly with USEMS, and to our knowledge this is the first case report describing migration of a USEMS in a proximal biliary obstruction. The advantages and disadvantages of various types of stents used for biliary obstruction and risk factors for stent migration are discussed.

\section{CASE REPORT}

A 52-year-old female presented to her general physician with a four-month history of right upper quadrant discomfort and was referred for an outpatient abdominal ultrasound scan. This revealed intrahepatic duct dilatation and subsequent computed tomography (CT) scan of liver and magnetic resonance cholangiopancreatography (MRCP) confirmed imaging features of a 24-mm intrahepatic cholangiocarcinoma. This was located immediately proximal to the confluence of the left and right hepatic ducts and was causing obstruction of the left intrahepatic ducts. There were no distant metastases identified on imaging or staging laparoscopy.

Endoscopic retrograde cholangiopancreatography (ERCP) and direct endoscopy of the bile duct was performed with the aim of directly visualizing the tumor to obtain a biopsy and confirm the diagnosis. Cannulation of the bile duct was difficult and a pre-cut sphincterotomy was performed in an attempt to facilitate access, but this was unsuccessful. The procedure was complicated by a duodenal perforation with retroperitoneal collection and pancreatitis, which delayed definitive management. The tumor was thought to be resectable after initial imaging, but unfortunately, repeat CT scan demonstrated progression over the subsequent weeks with involvement of the right portal vein and right intrahepatic ductal system.

The patient was discharged two weeks after the ERCP, but re-presented to the emergency department a week later complaining of abdominal pain and nausea. At this time, she was noted to be clinically jaundiced. Fluoroscopically guided percutaneous trans-hepatic cholangiography (PTC) and bare metal stent insertion was therefore performed under conscious sedation as a palliative procedure to relieve the obstruction. Following puncture of the right anterior ducts with an introducer set ('Accustick' introducer set, Boston scientific $®$ ), complete occlusion of the ductal system was demonstrated. There was no cross filling of the left segmental ducts. The obstruction was traversed with a guide wire. A $10 \times 70$ mm USEMS (Placehit Biliary WALLSTENT, Boston Scientific $($ ) was then deployed across the stricture and was positioned with $3 \mathrm{~cm}$ of stent proximal to the tumor to allow maximum proximal expansion within the duct and ensure optimal flow of bile from the intrahepatic ducts (Figure 1). Free flow of contrast into the common bile duct was demonstrated, indicating successful procedure and no balloon expansion was required.
The day after the procedure, the patient complained of abdominal pain and nausea, and developed a temperature of $38.5^{\circ} \mathrm{C}$. She was noted to be more jaundiced. Serum bilirubin rose from $84 \mathrm{mmol} / \mathrm{L}$ (normal range: 21-100 $\mathrm{mmol} / \mathrm{L}$ ) before the stent placement to $268 \mathrm{mmol} / \mathrm{L}$ two days post-procedure. Full blood count revealed elevated white cell count of $21 \times 10^{9} / \mathrm{L}$ (normal range: $4-11 \times 10^{9} / \mathrm{L}$ ) and serum C-reactive protein (CRP) increased to 47 $\mathrm{mg} / \mathrm{L}$ (normal range: $4-8 \mathrm{mg} / \mathrm{L}$ ). A diagnosis of biliary sepsis was made. Blood cultures grew Enterococcus faecium with resistance to amoxicillin. Teicoplanin, gentamycin and metronidazole were commenced. The liver function tests failed to improve over the next few days, and a repeat PTC was performed eight days after the first procedure which demonstrated that the stent had slipped through the stricture into the extra-hepatic biliary tree, and that the right intrahepatic duct was once again completely occluded (Figure 2). A wire was placed across the occlusion and through the centre of the migrated stent, and a further uncovered $10 x 70 \mathrm{~mm}$ selfexpandable metal stent (Placehit Biliary WALLSTENT, Boston Scientific $($ ) was then placed across the stricture

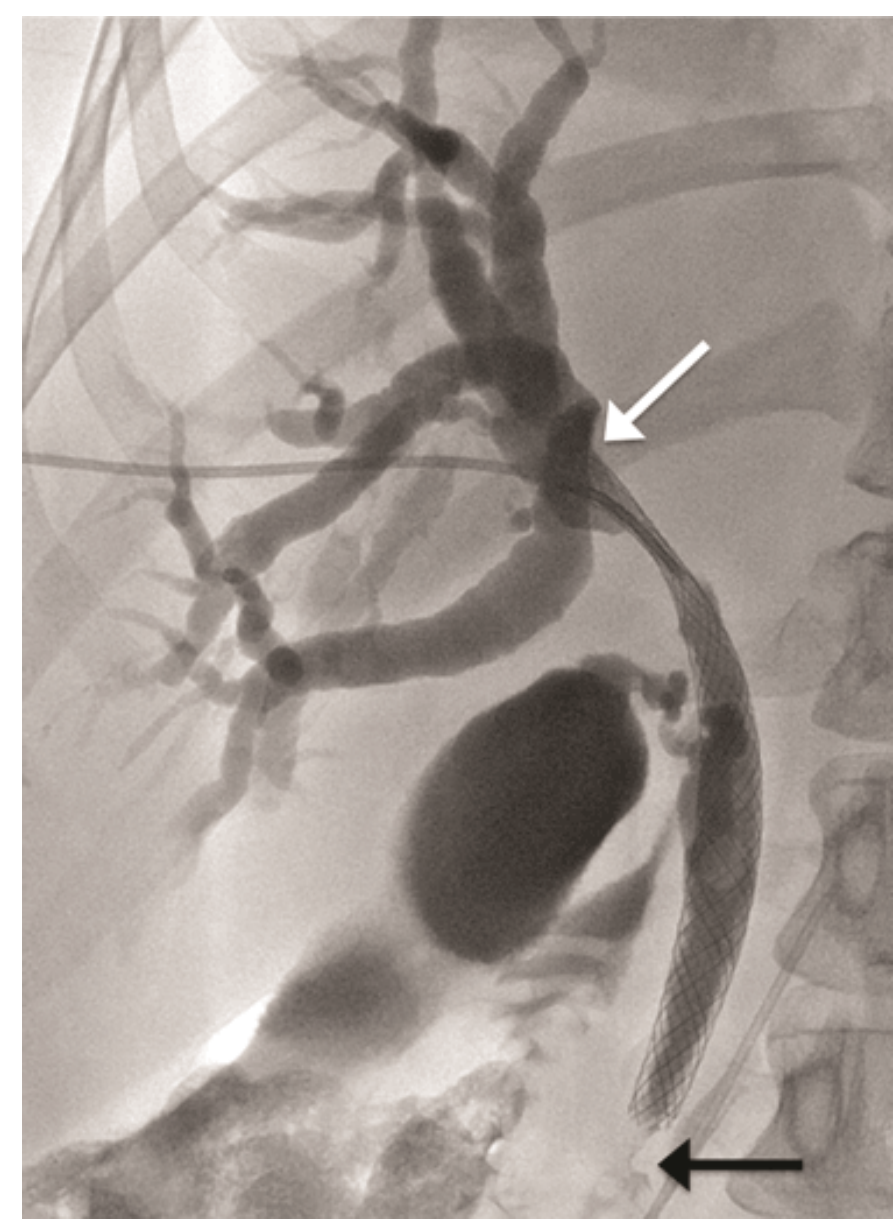

Figure 1: Uncovered self-expandable metal stent has been deployed across the stricture. The proximal aspect of the stent is shown at the confluence of the right hepatic ducts (white arrow). Contrast material flows freely from the distal aspect of the stent (black arrow), indicating successful procedure. 
and deployed, the distal end of the new stent sited within the proximal end of the migrated one. There was some wasting in the region of the tumor which was dilated using a balloon dilatation catheter (35LP Low-Profile PTA Balloon Dilatation Catheter (o.35”, 8 mm diameter $4 \mathrm{~cm}$ length). There was subsequent free flow of contrast into the duodenum indicating a successful procedure (Figure 3).

Following the placement of the second stent, the patient improved with reduction in pain and nausea. Serum bilirubin dropped from $195 \mathrm{mml} / \mathrm{L}$ to $119 \mathrm{mmol} / \mathrm{L}$ within two days, and she was discharged four days later for outpatient oncology follow-up.

\section{DISCUSSION}

\section{Plastic Versus Self-Expandable Metal Biliary Stents}

Compared with standard 1 of plastic stents, 10 $\mathrm{mm}$ self-expandable metal stents (SEMS) have an approximately three times greater transverse diameter which makes biliary obstruction less likely. One

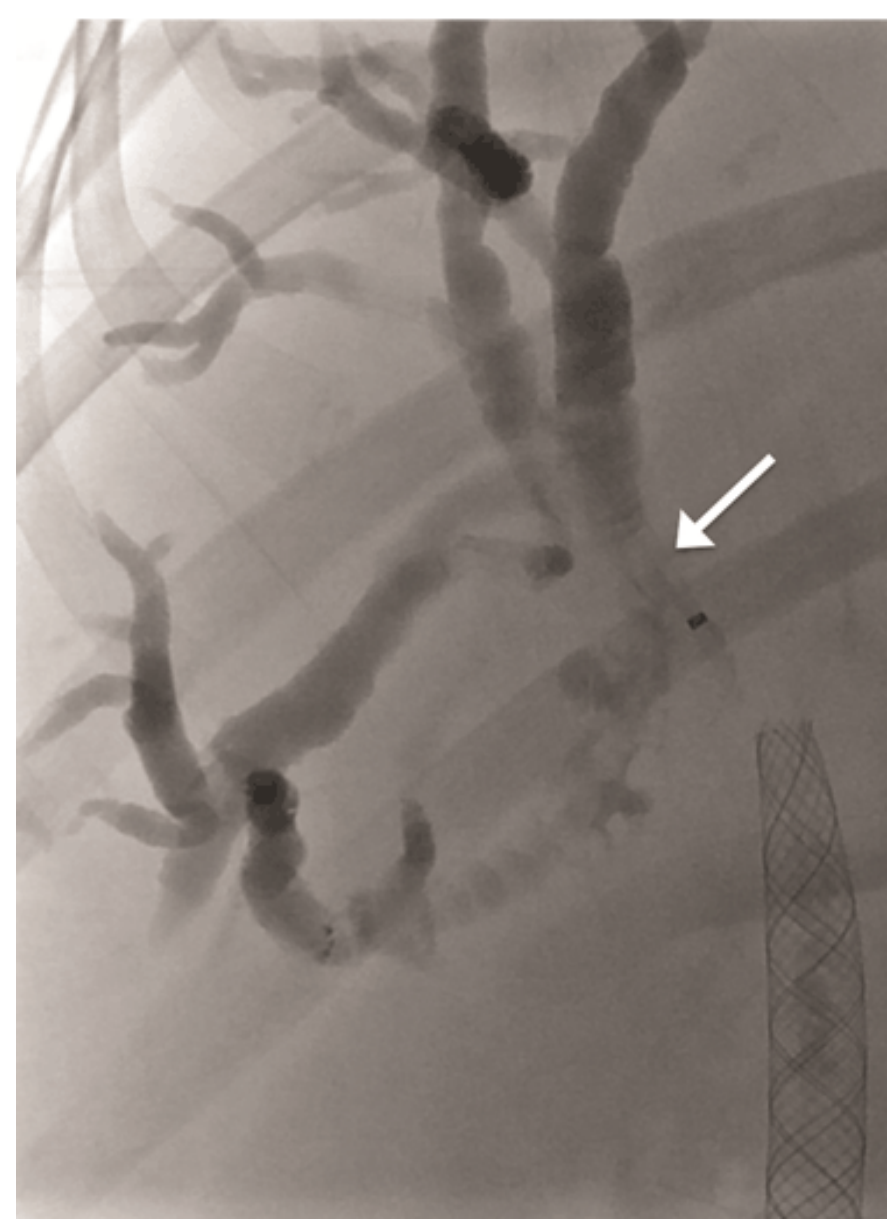

Figure 2: Right ductal system is once again obstructed. The stent has migrated from its original position (white arrow) at the confluence of the right hepatic ducts, and now lies beyond the stricture in the extrahepatic duct.

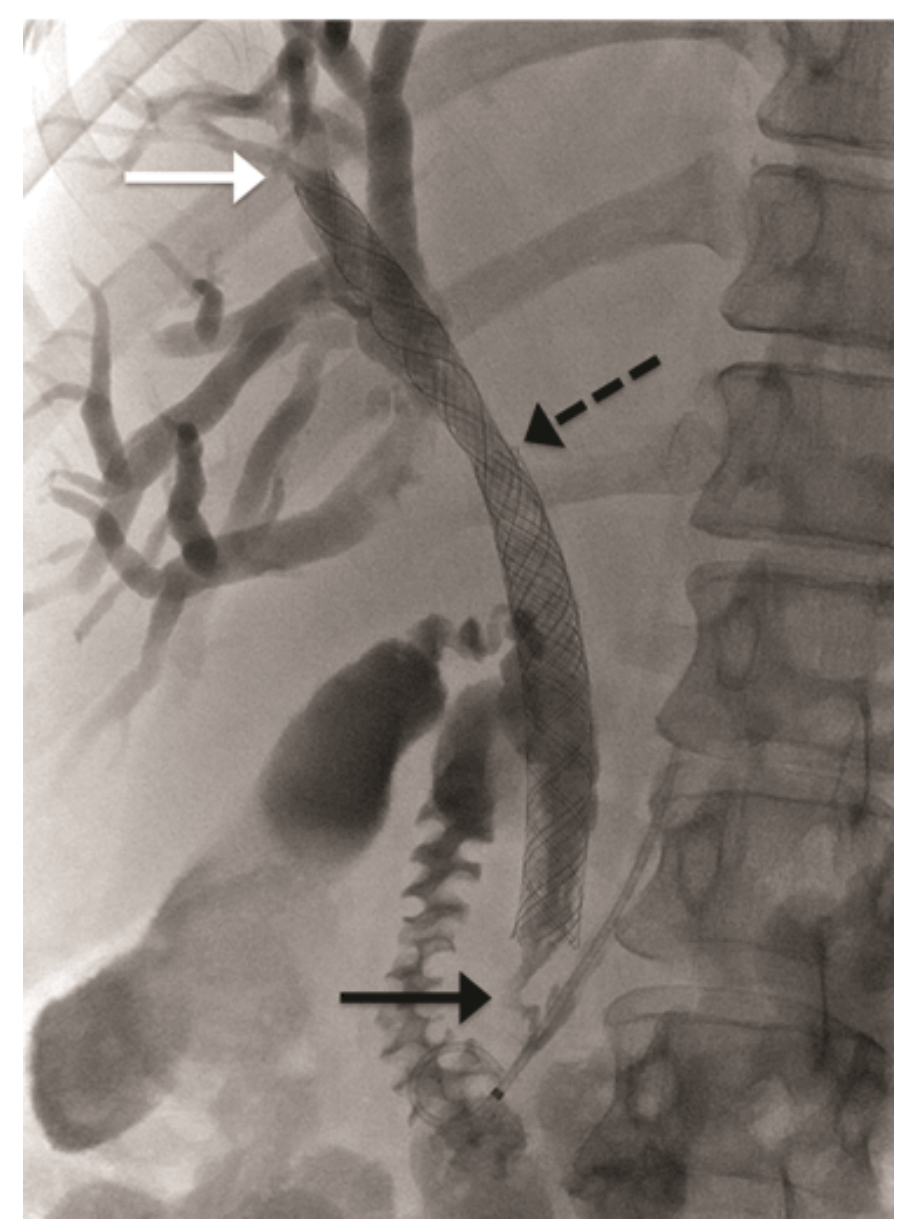

Figure 3: A second stent has been inserted, with proximal aspect well within a right segmental duct (white arrow) and distal aspect within the proximal end of the migrated stent (black broken arrow). Contrast material flows from the distal end of the original stent (black solid arrow), indicating successful procedure.

Cochrane review paper found no difference in technical success, therapeutic success, procedural complications or 30-day mortality between metal and plastic stents but found that metal stents lead to significantly less episodes of recurrent biliary obstruction, and reduced re-intervention rates [2]. A more recent meta-analysis comparing plastic versus metal stents found a longer patency, lower re-intervention rate, and longer mean survival in biliary obstruction treated with metal stents when compared to plastic stents [3]. This study also conducted a subgroup analysis comparing hilar versus distal biliary obstruction and reported a higher chance of stent occlusion in hilar obstruction, suggesting the use of a metal stent is preferable in these cases. Neither study looked specifically at the risks of stent migration.

\section{Covered Versus Uncovered Self-expand- able Metal Stents}

A number of factors may contribute to SEMS failure including tumor ingrowth, epithelial hyperplasia, and occlusion caused by debris or clot. This led to the 
development of covered SEMS which have a membrane that is intended to prevent penetration of cellular material through the stent wall. A meta-analysis of randomized controlled trials comparing CSEMS versus USEMS found that CSEMS were associated with significantly prolonged stent patency (weighted mean difference [WMD] 60.56 days; 95\% confidence interval [CI], 25.96, 95.17; $\mathrm{I}^{2}=$ o\%) and longer stent survival (WMD 68.87 days; 95\% CI, 25.64, 112.11; I[2] = 79\%). However, tumor overgrowth, sludge formation and most significantly stent migration were higher with CSEMS (relative risk [RR] 2.02; 95\% CI, 1.08, 3.78; I² = o\%), (RR 2.89; 95\% CI, 1.27, 6.55; $\mathrm{I}^{2}=$ o\%) (RR 8.11; 95\% CI, 1.47, 44.76; I² = o\%), [4].

It is well known that USEMS are extremely unlikely to migrate [5]. A randomized controlled trial comparing CSEMS versus USEMS by Telford et al. enrolled 129 patients between 2002 and 2008 and randomized patients into USEMS and CSEMS arms [6]. There was significantly more stent migration in the covered group with $8(12 \%)$ of the covered stents migrating compared with none of the uncovered stents (P.006). This, however, was principally for distal biliary strictures. In a prospective trial by Kullman et al. 400 patients were randomized to CSEMS or USEMS [7]. Stent migration occurred in six patients in the covered group versus no patients in the uncovered group. A retrospective review of 749 SEMS cases by Lee et al. [8] for distal biliary tract obstruction (578 USEMS and 171 CSEMS) found only three cases of migration in the USEMS group (0.5\%) and 12 cases $(7 \%)$ in the CSEMS group.

\section{Risk factors for stent migration}

Risk factors for migration of USEMS are not known as the numbers previously reported cases are small. However, there have been recent publications on the risks for migration of plastic stents and CSEMS. Yoshiaki Kawaguchi et al. [9] recently assessed the outcomes of 396 patients with bile duct stenosis who had undergone endoscopic plastic stent placement for a variety of indications (the majority for benign obstruction such as stones in the biliary tree), and found that distal biliary strictures and common bile duct diameters greater than 10 $\mathrm{mm}$ were risk factors for stent migration. They also found an increased risk of migration of straight plastic stents when compared with pigtail stents. A recent retrospective analysis of CSEMS migration conducted by Nakai et al. analyzed a number of patient and stent factors in 290 patients who had a CSEMS for distal biliary obstruction due to pancreatic malignancy [5]. Stent migration rate was $15.2 \%$, and significant risk factors for stent migration included chemotherapy, low radial force, and duodenal invasion by the pancreatic tumor.

Neither of these studies is directly relevant to our case in which an USEMS was deployed in a proximal location for a malignant stricture. Our patient underwent a sphincterotomy at ERCP to facilitate access, and had not had chemotherapy at time of stenting. Balloon expansion was not performed at the time of procedure. In their large retrospective review of 749 SEMS in malignant biliary obstruction, Lee et al. postulate that CSEMS are more likely to migrate because of their high axial force and because they do not embed in the adjacent tissue [8]. They also noted that two out of the three USEMS patients who had migration had undergone a sphincterotomy as was the case with our patient. However, it is notable that all of their patients had a distal biliary obstruction due to pancreatic malignancy, rather than proximal obstruction as in our case. Furthermore, previous studies have failed to show a difference in the risk of stent migration in patients who have undergone a biliary sphincterotomy [10] and balloon expansion is not always required for self-expanding stents. There is no literature describing a difference in patency or risk of migration between balloon expanded and non-balloon expanded SEMS.

\section{CONCLUSION}

Radiologist and endoscopist must be familiar with the relative advantages and disadvantages of the various types of stents available for relief of obstruction of the biliary tree, which are dependent on the aetiology and location of the obstruction, the duration that stent patency must be maintained for, and other patient factors. The risk factors for stent migration in plastic stents and covered self-expandable metal stents (CSEMS) described above may help when deciding on the most appropriate choice of stent and placement method. However, it is well established that uncovered self-expandable metal stents (USEMS) are extremely unlikely to migrate, and the risk factors for stent migration with USEMS are not known. To our knowledge, this is the first case report to describe migration of USEMS inserted for biliary obstruction caused by hilar cholangiocarcinoma. Further study of USEMS migration will help to determine the contributing causes of this uncommon complication.

$* * * * * * * * *$

\section{Author Contributions}

Edward Robert John Walton - Substantial contributions to conception and design, Acquisition of data, Drafting the article, Revising it critically for important intellectual content, Final approval of the version to be published Ewan Simpson - Substantial contributions to conception and design, Drafting the article, Final approval of the version to be published

Mark Callaway - Substantial contributions to conception and design, Revising it critically for important intellectual content, Final approval of the version to be published

\section{Guarantor}

The corresponding author is the guarantor of submission. 


\section{Conflict of Interest}

Authors declare no conflict of interest.

\section{Copyright}

(C) 2015 Edward Robert John Walton et al. This article is distributed under the terms of Creative Commons Attribution License which permits unrestricted use, distribution and reproduction in any medium provided the original author(s) and original publisher are properly credited. Please see the copyright policy on the journal website for more information.

\section{REFERENCES}

1. Andersen JR, Sørensen SM, Kruse A, Rokkjaer M, Matzen P. Randomised trial of endoscopic endoprosthesis versus operative bypass in malignant obstructive jaundice. Gut 1989 Aug;30(8):1132-5.

2. Moss AC, Morris E, Mac Mathuna P. Palliative biliary stents for obstructing pancreatic carcinoma. Cochrane Database Syst Rev 2006 Apr 19;(2):CDo04200.

3. Hong WD, Chen XW, Wu WZ, Zhu QH, Chen XR. Metal versus plastic stents for malignant biliary obstruction: An update meta-analysis. Clin Res Hepatol Gastroenterol 2013 Nov;37(5):496-500.

4. Saleem A, Leggett CL, Murad MH, Baron TH. Metaanalysis of randomized trials comparing the patency of covered and uncovered self-expandable metal stents for palliation of distal malignant bile duct obstruction. Gastrointest Endosc 2011 Aug;74(2):321-7.
5. Nakai $\mathrm{Y}$, Isayama $\mathrm{H}$, Kogure $\mathrm{H}$, et al. Risk factors for covered metallic stent migration in patients with distal malignant biliary obstruction due to pancreatic cancer. J Gastroenterol Hepatol 2014 Sep;29(9):1744-9.

6. Telford JJ, Carr-Locke DL, Baron TH, et al. A randomized trial comparing uncovered and partially covered self-expandable metal stents in the palliation of distal malignant biliary obstruction. Gastrointest Endosc 2010 Nov;72(5):907-14.

7. Kullman E, Frozanpor F, Söderlund C, et al. Covered versus uncovered self-expandable nitinol stents in the palliative treatment of malignant distal biliary obstruction: Results from a randomized, multicenter study. Gastrointest Endosc 2010 Nov;72(5):915-23.

8. Lee JH, Krishna SG, Singh A, et al. Comparison of the utility of covered metal stents versus uncovered metal stents in the management of malignant biliary strictures in 749patients. Gastrointest Endosc 2013 Aug;78(2):312-24.

9. Kawaguchi Y, Ogawa M, Kawashima Y, et al. Risk factors for proximal migration of biliary tube stents. World J Gastroenterol 2014 Feb 7;20(5):1318-24.

10. Güitrón $\mathrm{A}$, Adalid $\mathrm{R}$, Barinagarrementería $\mathrm{R}$, Gutiérrez-Bermúdez JA, Martínez-Burciaga J. Incidence and relation of endoscopic sphincterotomy to the proximal migration of biliary prostheses. Rev Gastroenterol Mex 2000 Oct-Dec;65(4):159-62. [Article in Spanish].
Access full text article on other devices

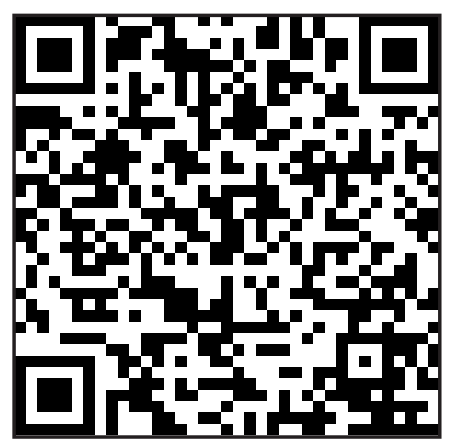

Access PDF of article on other devices

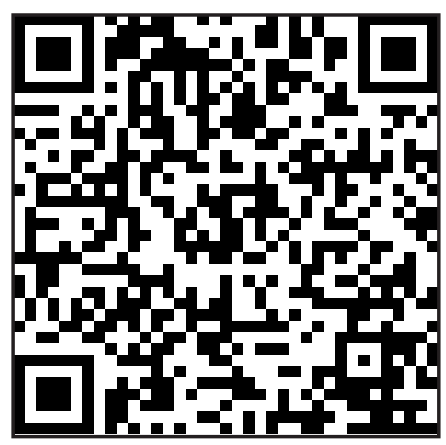

